\title{
Transcriptome screen for fast evolving genes by Inter-Specific Selective Hybridization (ISSH)
}

\author{
Juan I Montoya-Burgos", Aurélia Foulon, Ilham Bahechar
}

\begin{abstract}
Background: Fast evolving genes are targets of an increasing panel of biological studies, from cancer research to population genetics and species specific adaptations. Yet, their identification and isolation are still laborious, particularly for non-model organisms. We developed a method, named the Inter-Specific Selective Hybridization (ISSH) method, for generating CDNA libraries enriched in fast evolving genes. It utilizes transcripts of homologous tissues of distinct yet related species. Experimental hybridization conditions are monitored in order to discard transcripts that do not find their homologous counterparts in the two species sets as well as transcripts that display a strong complementarity between the two species. Only heteroduplexes that disanneal at low stringency are used for constructing the resulting cDNA library.

Results: We demonstrate the efficiency of the ISSH method by generating a brain CDNA library enriched in fast evolving transcripts of a non-model catfish species as well as a control, non-enriched library. Our results indicate that the enriched library contains effectively more fast evolving sequences than the control library. Gene annotation analyses also indicate enrichment in genes with low expression levels and non-ubiquitously expressed genes, both categories encompassing the majority of fast evolving genes. Furthermore, most of the identified transcripts show higher sequence divergence between two closely related catfish species as compared to recognized fast evolving DNA markers.

Conclusions: The ISSH method offers a simple, inexpensive and efficient way to screen the transcriptome for isolating fast evolving genes. This method opens new opportunities in the investigation of biological mechanisms that include fast evolving genes, such as the evolution of lineage specific processes and traits responsible for species adaptation to their environment.
\end{abstract}

\section{Background}

Fast evolving DNA sequences are used for answering a broad range of biological questions relative to population processes and phylogeography [e.g. [1]], species diversification [e.g. [2,3]], conservation biology [4] and also genome or phenotype mapping [e.g. [5]]. However, due to the very same intrinsic quality for which they are looked for, i.e. their high evolutionary rate, fast evolving DNA sequences display "lineage specific" changes and therefore require de novo development each time a new group of non-model organisms is being investigated. Despite various methodologies targeted toward the isolation of unspecific polymorphic DNA fragments [e.g. [6-8]] the identification and the isolation of fast evolving

\footnotetext{
* Correspondence: juan.montoya@unige.ch

Department of Zoology and Animal Biology, University of Geneva, 30 quai Ernest Ansermet, 1211 Geneva 4, Switzerland
}

DNA sequences in non-model organisms is still laborious and expensive, making it a major impediment to the routine analysis of multiple loci on many taxa.

The isolation of fast evolving genes has gained new motivation and attention as genes involved in several actively investigated processes display high substitution rates: the evolution of species specific traits such as the human brain [e.g. $[9,10]]$, speciation genes [e.g. $[11,12]]$, reproduction genes [e.g. $[13,14]]$ or genes governing the evolution of adaptive traits [e.g. [15]]. Theoretical approaches suggest that adaptation genes should be fast evolving so that selection could have a substrate on which to act [16]. Furthermore, speciation genes, those that are directly or indirectly involved in the establishment of the genetic barrier between closely related species, consistently displayed high divergence rates [11]. At present, fast evolving genes which often evolve under 
positive selection can be identified either through large genomic comparisons which are feasible only for model organisms like Drosophila species [e.g. $[17,18]]$ or human-chimpanzee comparisons [19] or via long term experimental approaches such as in the discovery of the hybrid inviability gene $\mathrm{Hmr}$ in Drosophila [20]. The increasing interest in biological mechanisms driven by fast evolving genes appeals to the development of a more efficient and cost effective method for the isolation of such genes across closely related species and which would not imply the prior knowledge of genetic or genomic information.

Here we describe a simple and efficient experimental approach for enriching a cDNA library in fast evolving transcript fragments. Our method, named Inter-Specific Selective Hybridization (ISSH) is based primarily on the principles of the widely used subtractive hybridization $(\mathrm{SH})$ procedure developed more than two decades ago for isolating cDNAs of differentially expressed genes [21-23]. In the original $\mathrm{SH}$ protocol, the hybridization of cDNAs versus mRNAs of different cell lines belonging to the same organism distinguishes transcripts that are equally expressed in both cell lines from those that are uniquely or differentially expressed in the cell line of interest. In the ISSH approach (Figure 1), the transcriptome of the species of interest ("probed" species) is reverse transcribed into single stranded (ss) cDNA and then hybridized against the biotinylated mRNA pool of a distinct yet close relative ("selector" species). During hybridization, three populations of transcripts of the species of interest can be found: (1) transcripts that never find their selector counterparts due to differential expression or gene loss; (2) fast evolving transcripts that find their homologous counterparts but the resulting heteroduplexes are unstable due to numerous nucleotide mispairings; and (3) conserved transcripts that form stable heteroduplexes. The second population of transcripts is in fact composed by sequences of varying divergence. The transcripts of interest are those that disanneal at the lowest stringencies and are therefore rescued for constructing the cDNA library enriched in fast evolving transcripts. The ISSH method was applied for isolating fast evolving transcript fragments of the nonmodel catfish species Ancistrus temminckii (family Loricariidae). We used the zebrafish genome as a reference for assessing the sequence divergence of the isolated transcripts and for transcript annotation and characterization.

\section{Results}

\section{Experimental design}

The ISSH method (Figure 1) confronts in solution complementary transcriptomes of two closely related species with the aim of rescuing transcripts of fast evolving

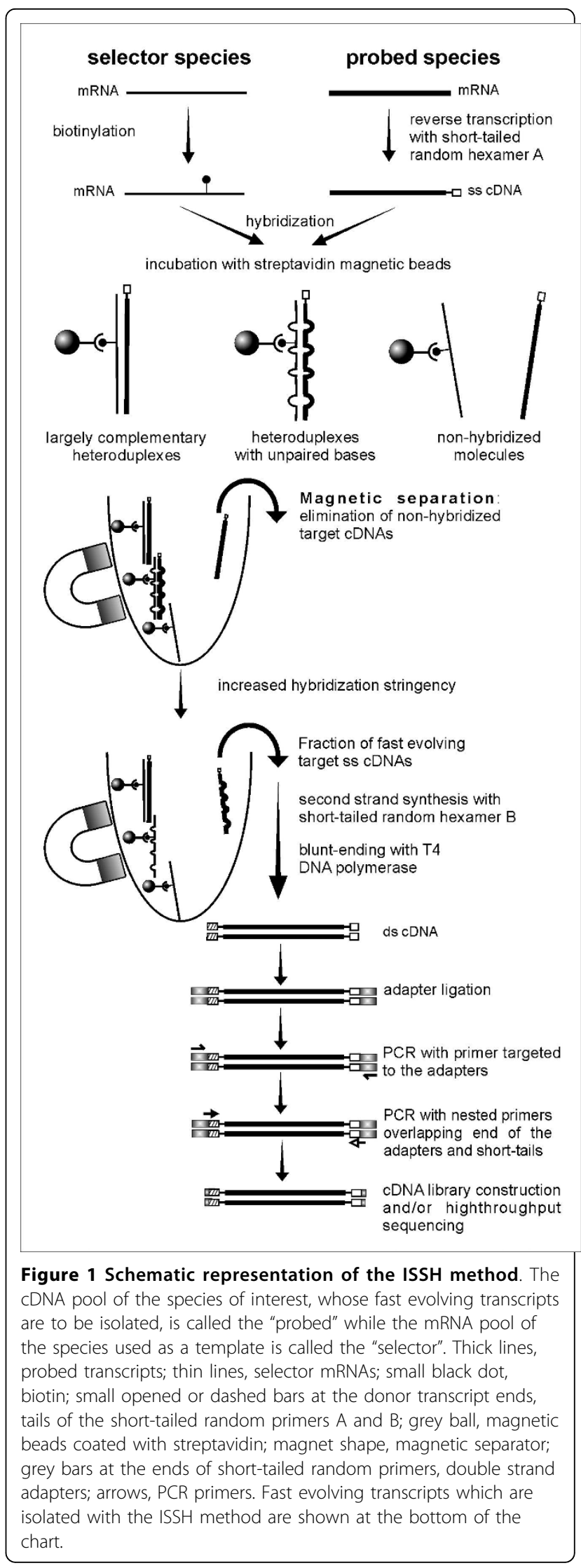


genes. The property of evolving fast implies that such transcripts will disanneal at low stringencies from the heteroduplexes formed by homologous complementary sequences of the two species. Our method was applied to build a cDNA library enriched in fast evolving transcript fragments of brain tissue of the catfish Ancistrus temminckii. We used as the selector species its close relative Ancistrus dolichopterus. To assess the efficiency of the ISSH method we prepared a non-enriched control cDNA library of brain tissue of $A$. temminckii using standard protocols. The two libraries were sequenced with the FLX Genome Sequencer technology (Roche). We then "blasted" the enriched and control libraries against the complete genome of the zebrafish and analyzed the differences. We also annotated the transcripts producing significant matches and examined their characteristics to highlight the effectiveness of our method. As the zebrafish is not a close relative to our catfish and because the sequences of interest display high sequence divergence, a substantial proportion of the enriched library yielded no significant Blast matches. Therefore, we prepared an EST library of a close catfish relative, Hypostomus gr. plecostomus, belonging to the same subfamily (Loricariidae: Hypostominae), for refining the analyses.

\section{Analysis of sequence divergence}

High-throughput sequencing and reads assembly yielded 2429 and 1255 contigs for the enriched and control libraries respectively. We blasted the contigs against the zebrafish genome using parameters suitable for comparing divergent sequences. Only Blast results with E-values lower than $10 \mathrm{e}-5$ were considered for the analyses; they represented $45.7 \%$ and $40.6 \%$ of all contigs of the enriched and control libraries, respectively. Among the contigs producing non-significant Blast matches about half were low-complexity sequences as indicated by RepeatMasker and were excluded from further analyses (20.7\% and $22 \%$ of the enriched and control libraries, respectively). Significant Blast alignments were classified by size in order to produce a finer analysis of the differences that exist between the enriched and control libraries. The sequence divergence comparisons (Table 1) consistently show that the enriched library displays more divergent sequences (higher mean) than the control library using as reference the zebrafish genome. Applying a mean t-test unambiguously indicates that the mean divergence per category is significantly higher in the enriched library than in the control library, and this for all size categories (Table 1 ).

When using the zebrafish genome as reference, the fastest evolving sequences may not find their homologous counterparts due to the distant evolutionary relationship between the zebrafish and our non-model catfish. Thus, performing the same analysis yet using an evolutionary closer reference - our EST database of the catfish Hypostomus gr. plecostomus - may allow a better understanding of the efficiency of ISSH method. The sequence

Table 1 Analysis of sequence divergence for the enriched and the control libraries.

\begin{tabular}{|c|c|c|c|c|c|c|}
\hline \multirow{2}{*}{$\begin{array}{l}\text { Size category } \\
\text { (bp.) }\end{array}$} & \multicolumn{2}{|c|}{ Enriched library } & \multicolumn{2}{|c|}{ Control library } & \multirow[t]{2}{*}{ T-test (df-t) } & \multirow[t]{2}{*}{$P$} \\
\hline & Mean (S.D.) & $\mathrm{n}$ & Mean (S.D.) & $\mathrm{n}$ & & \\
\hline \multicolumn{7}{|c|}{ Blast against zebrafish } \\
\hline 90-109 & $29.93(7.56)$ & 126 & $26.93(8.77)$ & 66 & $-2.358(116)$ & $0.010^{*}$ \\
\hline $110-129$ & $33.32(9.26)$ & 129 & $28.84(10.06)$ & 67 & $-3.038(124)$ & $0.001^{* *}$ \\
\hline $130-149$ & $34.82(9.86)$ & 138 & 31.61 (11.06) & 61 & $-1.95(103)$ & $0.027^{*}$ \\
\hline $150-169$ & $36.84(9.83)$ & 103 & $33.56(11.43)$ & 47 & $-1.701(78)$ & $0.046^{*}$ \\
\hline $170-189$ & $40.42(9.88)$ & 100 & $33.34(14.11)$ & 36 & $-2.777(47)$ & $0.004^{* *}$ \\
\hline $190-209$ & 40.97 (10.01) & 94 & 37.03 (13.14) & 39 & $-1.681(57)$ & $0.049^{*}$ \\
\hline $210-229$ & $40.52(10.92)$ & 80 & 28.91 (14.08) & 55 & $-5.143(96)$ & $<0.001^{* *}$ \\
\hline $230-249$ & $39.33(11.01)$ & 64 & 34.63 (13.87) & 34 & $-1.71(55)$ & $0.045^{*}$ \\
\hline$\geq 250$ & $42.39(11.41)$ & 145 & $34.72(15.60)$ & 34 & $-2.702(41)$ & $0.005^{* *}$ \\
\hline \multicolumn{7}{|c|}{ Blast against Hypostomus catfish } \\
\hline $90-109$ & $30.09(9.93)$ & 131 & $24.70(12.86)$ & 66 & $-2.986(105)$ & $0.002^{* *}$ \\
\hline $110-129$ & $31.66(11.29)$ & 140 & $22.12(12.62)$ & 49 & $-4.771(78)$ & $<0.001^{* *}$ \\
\hline $130-149$ & $35.30(12.17)$ & 127 & $27.48(12.30)$ & 33 & $-3.261(49)$ & $<0.001^{* *}$ \\
\hline $150-169$ & $36.18(12.55)$ & 115 & $29.40(15.87)$ & 39 & $-2.423(55)$ & $0.009^{* *}$ \\
\hline $170-189$ & $37.93(11.71)$ & 96 & $31.87(13.66)$ & 19 & $-1.807(23)$ & $0.04^{*}$ \\
\hline $190-209$ & $36.53(12.15)$ & 103 & $27.73(12.41)$ & 25 & $-3.193(36)$ & $0.001^{* *}$ \\
\hline $210-229$ & $43.66(12.01)$ & 48 & $28.76(15.48)$ & 18 & $-3.689(25)$ & $<0.001^{* *}$ \\
\hline$\geq 230$ & $36.16(12.08)$ & 99 & $28.44(18.10)$ & 18 & $-1.740(19)$ & $0.047^{*}$ \\
\hline
\end{tabular}


divergence comparisons (Table 1) show again a systematic and significant enrichment in fast evolving sequences in the enriched library as compared to the control library. The difference between the two libraries is generally higher than when using the zebrafish as reference. This is likely explained by the inclusion of a set of faster evolving genes which can now find their homologues in the evolutionary closer Hypostomus reference.

\section{Characteristics of the sequences retained by the ISSH method}

In order to better assess the usefulness of the ISSH method we annotated the contigs of the enriched and control libraries according to the information collected from their translated best Blast hit in the Swissprot/Uniprot database with a minimum threshold of E-score $\leq$ $10 \mathrm{e}-8$. In this way only 60 contigs were characterized in the enriched library (2.5\% of all contigs) and 39 in the control library (3.1\% of all contigs). The analysis of the annotated sequences will serve to test three predictions that have to be fulfilled if the method achieves its goal. First, as mitochondrial genes evolve significantly faster than the vast majority of nuclear genes they should be more numerous in the enriched library than in the control. The results indicate that mitochondrial genes represent $22.5 \%$ versus $8.6 \%$ of the annotated contigs of the enriched and control libraries, respectively, fulfilling the prediction. The second expectation concerns the overall correlation between the expression level and gene sequence conservation, where conserved genes are generally expressed at higher rates than fast evolving genes [24]. The expression level of the annotated contigs were approximated by using Unigene database information on the expression level of their orthologous genes in nervous system tissues of the zebrafish or, alternatively, of the mouse or human when the data was not available. Annotated contigs were classified into four categories of gene expression levels (Figure 2). We discarded here the mitochondrial genes which are fast evolving yet possess a high expression level. As expected, the enriched library is essentially composed by genes belonging to the category with the lowest expressionlevel (58\% of the total versus $26 \%$ in the control library). The enriched library also shows a depletion of genes in the highly expressed gene categories, which are generally the more conserved ones. The third prediction refers to the observation that genes with tissue-specific expression evolve generally faster than genes with ubiquitous expression, a category in which most housekeeping genes are found [25,26]. As predicted, our method resulted in an enrichment of non-ubiquitously expressed genes totaling $57 \%$ of the annotated contigs of the enriched library versus $35 \%$ in the control library. The non-ubiquitously expressed genes are defined as

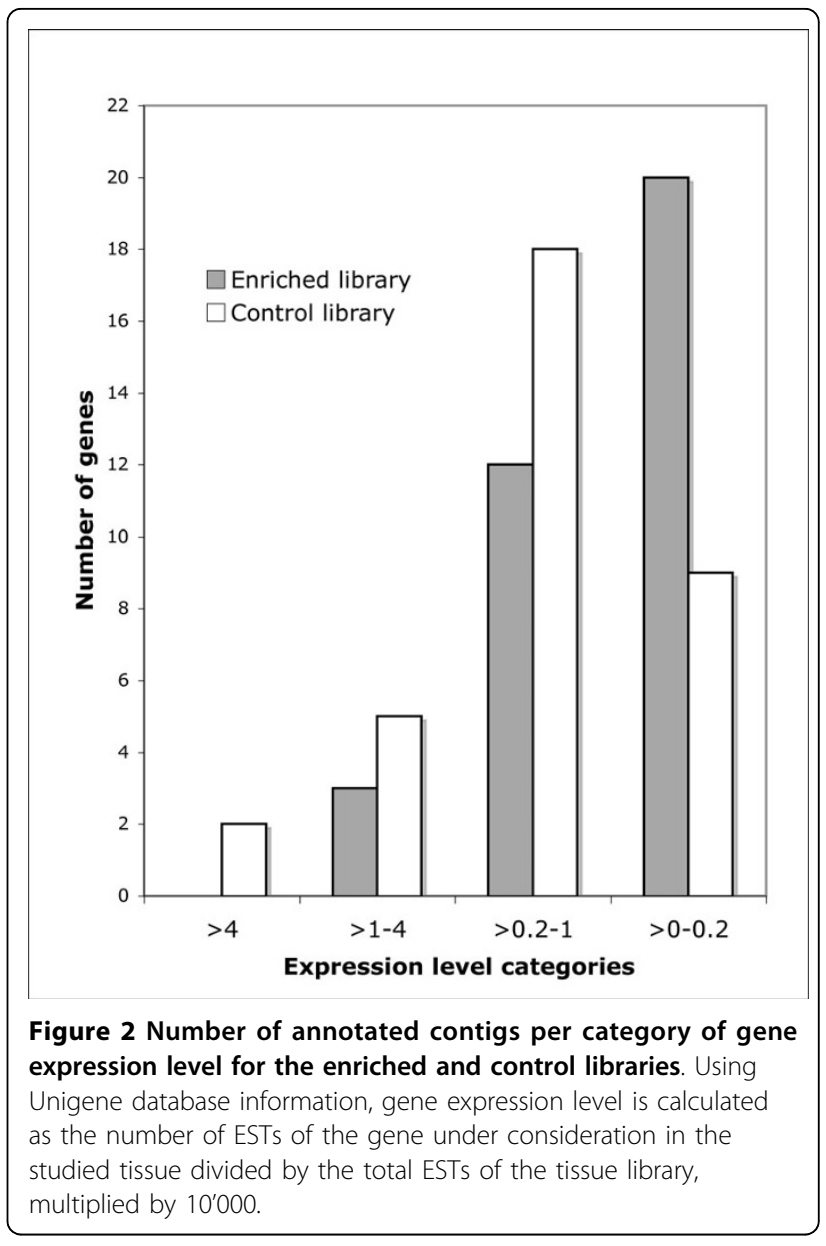

those expressed in less than four tissues according to Unigene expression information.

In an attempt to better characterize the fast evolving transcripts isolated in Ancistrus temminckii and which show a putative orthologous sequence in the Hypostomus gr. plecostomus EST dataset, we search for a tentative annotation by Blastn comparisons against the mRNA reference sequences (mRNA refseq) database of NCBI, limited to teleost sequences (a threshold of $\mathrm{E}<$ $1 \mathrm{e}-8$ was used). In this way, 26 transcripts present in the catfishes Ancistrus and Hypostomus, as well as in the teleost mRNA refseq database were annotated and their sequence divergence was calculated based on the overlapping region of the sequence alignment, allowing a direct comparison (Table 2). The tentatively annotated transcript fragments show an overall high sequence divergence between the two Hypostominae catfishes (mean $=0.28 \pm 0.09)$, not much different from the divergence between Ancistrus and the closest teleost orthologous sequence of the mRNA refseq database (mean $=0.39 \pm 0.08)$, which do not include catfish sequences. Interestingly, about half of the transcript fragments encompasses coding sequence (cds) (Table 2). 
Table 2 Tentatively annotated fast evolving transcript fragments and their sequence divergence as compared to the closest ortholog in the teleost mRNA refseq database and in the Hypostomus gr. plecostomus EST dataset.

\begin{tabular}{|c|c|c|c|c|c|}
\hline contig & mRNA refseq annotation according to closest teleost ortholog & Species & $\begin{array}{l}\text { Cds/ } \\
\text { UTR }\end{array}$ & $\begin{array}{c}\text { A.temminckii vs } \\
\text { closest teleost ortholog } \\
\text { in mRNA refseq }\end{array}$ & $\begin{array}{l}\text { A. temminckii vs } H \text {. } \\
\text { gr. plecostomus }\end{array}$ \\
\hline 342 & zgc:175146 & Dr & 3'UTR & 0.4411 & 0.2990 \\
\hline 435 & interferon regulatory factor 6 (irf6) & Dr & 3'UTR & 0.4824 & 0.3485 \\
\hline 478 & $\begin{array}{l}\text { NADH dehydrogenase (ubiquinone) } 1 \text { alpha subcomplex, assembly } \\
\text { factor } 2 \text { (ndufaf2), nuclear gene encoding mitochondrial protein }\end{array}$ & Dr & 3'UTR & 0.2680 & 0.2531 \\
\hline 597 & similar to porcupine homolog (LOC100148644) & Dr & 3'UTR & 0.4824 & 0.3485 \\
\hline 605 & zgc:158374 & Dr & cds & 0.4411 & 0.2833 \\
\hline 710 & single-minded homolog $2(\operatorname{sim} 2)$ & Dr & 3'UTR & 0.4411 & 0.3151 \\
\hline 785 & similar to pol polyprotein (LOC796496) & Dr & cds & 0.3839 & 0.1324 \\
\hline 809 & similar to ORF1-encoded protein (LOC100004717) & Dr & 5'UTR & 0.4824 & 0.3839 \\
\hline 1137 & zgc:56382 & Dr & cds & 0.3485 & 0.2680 \\
\hline 1451 & RMD5 homolog B (rmd5b) & Ss & 5'UTR & 0.5042 & 0.1324 \\
\hline 1479 & similar to ORF1-encoded protein (LOC100004764) & Dr & 3'UTR & 0.2531 & 0.1573 \\
\hline 1492 & wu:fc33e05 & Dr & 3'UTR & 0.3151 & 0.2680 \\
\hline 1565 & hypothetical LOC570897 & Dr & cds & 0.3316 & 0.2385 \\
\hline 1614 & $\begin{array}{l}\text { ras-related C3 botulinum toxin substrate } 1 \text { (rho family, small GTP } \\
\text { binding protein Rac1) like (rac1l) }\end{array}$ & Dr & 3'UTR & 0.2833 & 0.2990 \\
\hline 1694 & similar to NLR family, pyrin domain containing 3 (LOC100002061) & Dr & 3'UTR & 0.3839 & 0.4024 \\
\hline 1695 & monoacylglycerol O-acyltransferase 2 & Dr & 3'UTR & 0.3485 & 0.2531 \\
\hline 1782 & similar to G protein-coupled receptor 128 (LOC100148710) & Dr & cds & 0.3839 & 0.2531 \\
\hline 1819 & $\begin{array}{l}\text { similar to Uromodulin precursor (Tamm-Horsfall urinary glycoprotein) } \\
\text { (THP) (LOC100007639) }\end{array}$ & Dr & cds & 0.4824 & 0.4024 \\
\hline 1902 & hypothetical protein LOC100150258 & Dr & cds & 0.3839 & 0.1324 \\
\hline 1905 & si:dkeyp-27b10.2 & Dr & cds & 0.3485 & 0.2531 \\
\hline 2016 & zgc:64076 & Dr & 3'UTR & 0.2680 & 0.1702 \\
\hline 2029 & zgc:85811 & Dr & cds & 0.3151 & 0.1833 \\
\hline 2066 & similar to CG6639 CG6639-PA (LOC100000002) & Dr & cds & 0.4824 & 0.3151 \\
\hline 2085 & hypothetical protein LOC100149782 & Dr & cds & 0.2990 & 0.2103 \\
\hline 2225 & zgc:158374 & Dr & cds & 0.4614 & 0.4214 \\
\hline \multirow[t]{5}{*}{2342} & similar to zymogen granule membrane glycoprotein 2 (LOC100005977) & Dr & cds & 0.4614 & 0.4411 \\
\hline & Reference fast evolving sequences & & & $\begin{array}{l}\text { A. brevipinnis vs Danio } \\
\text { rerio }\end{array}$ & $\begin{array}{l}\text { A. brevipinnis vs } \mathrm{H} \text {. } \\
\text { boulengeri }\end{array}$ \\
\hline & cytochrome oxidase subunit I (COI) & & cds & 0.239 & 0.145 \\
\hline & & & & A. cirrhosus vs Danio rerio & $\begin{array}{l}\text { A. cirrhosus vs } \mathrm{H} \text {. } \\
\text { boulengeri }\end{array}$ \\
\hline & reticulon 4 (RTN4) introns $1 \& 2$ & & introns & 0.748 & 0.170 \\
\hline
\end{tabular}

Sequence divergence was calculated using the alignment region with sequence in all species compared. Divergences were corrected using the K2P model with 2 transitions per transversion. The lowest part of the table presents the sequence divergence of two published fast evolving markers used for characterizing species or genera. Dr: Danio rerio; Ss:

Salmo salar; A.: Ancistrus; H.:Hypostomus.

To estimate whether the sequence divergence is higher than in recognized fast evolving coding and non-coding DNA markers, we calculated the sequence divergence of the mitochondrial cytochrome oxydase I gene (COI), used by the Barcode of Life Initiative for characterizing species http://www.dnabarcodes.org/, and the two introns of the reticulon 4 (RTN4) gene, used to infer fish phylogenies at the specific level [2]. Most of the tentatively annotated transcripts display higher sequence divergence between Ancistrus temminckii and Hypostomus gr. plecostomus, as compared to the two reference markers (Table 2), validating once again the ISSH method. Likewise, most of the transcripts show higher sequence divergence between Ancistrus temminckii and Danio rerio as compared to the COI sequence. However, the intronic sequences of the RTN4 are much more divergent than the annotated transcripts. This is likely explained by the frequent insertion/deletion events in non-coding sequences and which enhance drastically the sequence divergence between distantly related species.

We emphasize that the sequences of the transcripts annotated using the mRNA refseq database likely 
represent the most conserved regions of the isolated transcripts dataset, as faster evolving regions will not find their sequence counterparts in the refseq database, which comprises no closely related catfish sequences.

\section{Discussion}

The isolation of fast evolving genes can be easily accomplished on model organisms for which abundant genomic and transcriptomic knowledge exist. Bioinformatic routines and experimental procedures (micro-array technology) are available for this purpose. At present, however, there is no efficient method for doing so in nonmodel organisms. The ISSH method presented here is a fast and cost-effective procedure for enriching a cDNA library in fast evolving genes. The various tests we have performed resulted in a convincing demonstration of the efficiency of our method. We have shown that the overall sequence divergence was significantly increased in the enriched library as compared to the control when blasting these libraries against the zebrafish genome or against our Hypostomus catfish EST library. Moreover, the results of the ISSH method fulfilled the three predictions made upon the knowledge of general properties of fast versus slowly evolving genes. Briefly, the enriched library displayed (1) a higher proportion of fast evolving mitochondrial genes, (2) a higher fraction of genes with low expression level, and (3) proportionally more nonubiquitously expressed genes. Furthermore, the fast evolving transcripts with orthologous sequences in the two catfish species and in the mRNA refseq fish database displayed generally higher sequence divergence than recognized fast evolving DNA markers.

A complementary support of these results comes from the Gene Ontology (GO) classification. The GO annotation (Figure 3) showed that genes involved in metabolic processes were less abundant in the enriched library than in the control (30\% versus $64 \%$ of all annotated contigs in the enriched and control libraries, respectively). This is consistent with the observation that the set of housekeeping genes is generally rich in metabolic genes, for example in human [27]. Besides indicating that the enriched library is depleted in housekeeping genes, the GO annotation also shows that this library is enriched in nervous system tissue-specific genes (Neuronal activity, Figure 3), highlighting once more the ability of the method in isolating genes with tissue-specific expression that generally evolve faster than ubiquitously expressed genes.

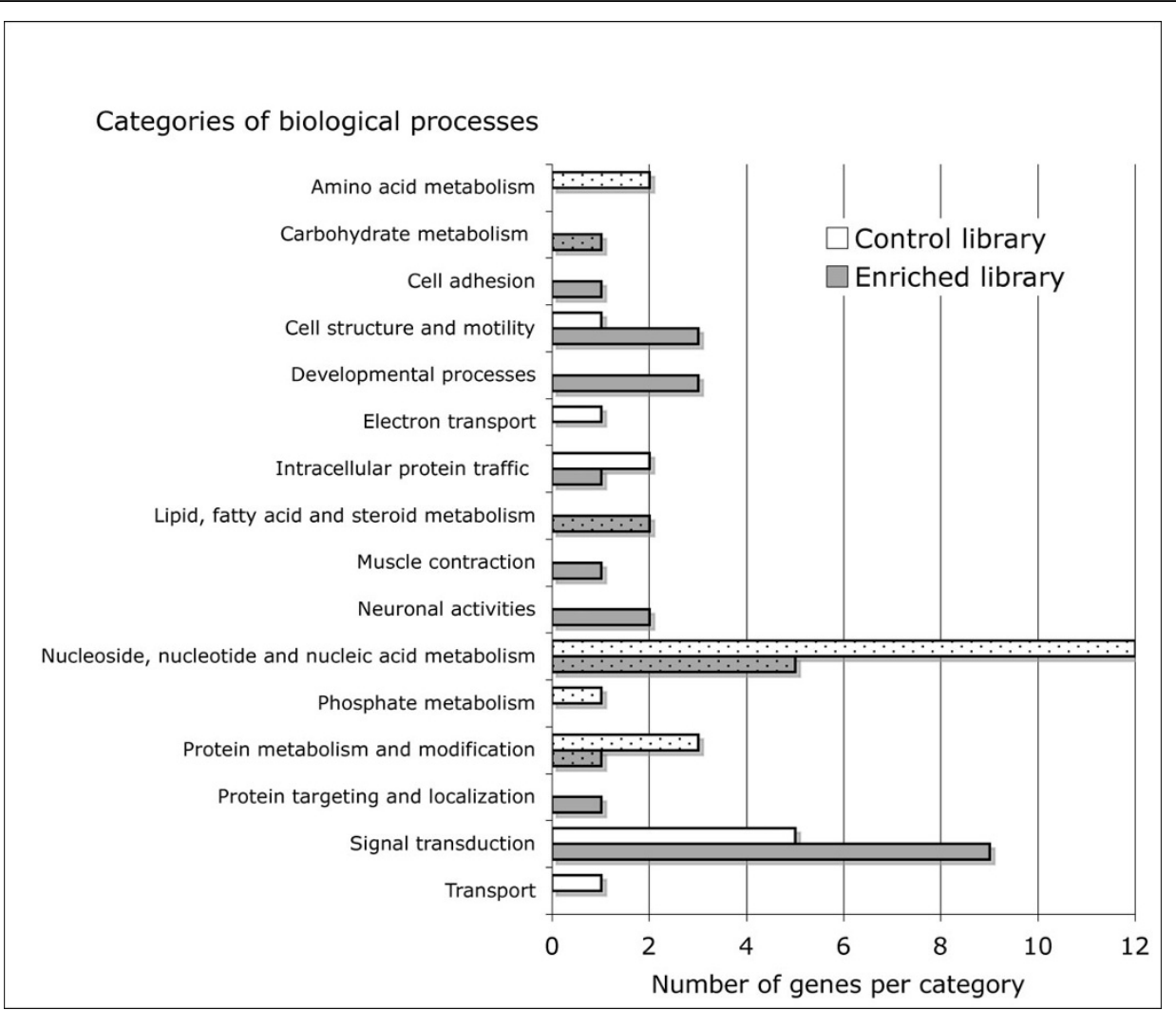

Figure 3 Gene ontology classification of the fraction of annotated transcripts belonging to the library enriched in fast evolving genes and the control library. Only the major categories of biological processes are used, according to Panther database. Dotted bars indicate biological processes involved in metabolism. 
The proportion of annotated contigs via Uniprot/Swissprot comparisons is rather small, particularly in the enriched library. This can be explained firstly by the relatively poor representation of fish genes in the Uniprot/Swissprot database combined with the likely high sequence divergence between the genes of the non-fish organism in the database and our catfish. Secondly, not all contigs may contain coding sequence; they may be composed mainly of UTR sequence. However, the enriched library shows no marked bias toward UTR sequences, which evolved generally faster than their contiguous coding sequences. Indeed, about $68 \%$ of contigs longer that $240 \mathrm{bp}$ display putative open reading frames (ORF) longer than 80 aa (criterion of the $\mathrm{H}$-invitational annotation project), and $51 \%$ of contigs longer that $300 \mathrm{bp}$ display putative ORFs longer than 100 aa (criterion of the Functional Annotation of Mouse (FANTOM) project), which corresponds to four and five times the calculated sequence length without stop codons in non-coding sequence using the same base frequencies, respectively. Similar proportions are observed in the control library (70\% and 54\%, respectively) indicating no strong enrichment in UTR sequences. Furthermore, a significant part of the isolated transcripts may be non-coding RNAs. It has been shown, for instance, that non-coding RNAs constitute more than half of the mammalian transcriptome [28]. As the annotation of the isolated fast evolving transcripts is difficult due to the lack of sequence similarity with distant reference species, we are unable for the moment to assess the proportion of fast evolving non-coding RNAs in our dataset.

Our method has the advantage of being theoretically very versatile in terms of evolutionary divergence relating the species of interest and its selector species. The faster evolving genes will already show detectable sequence divergence between closely related species while using a more distant selector species will allow the isolation of a wider set of fast evolving genes. Likewise, one can modify the hybridization temperature to fine-tune the degree of sequence divergence one is looking for between the species of interest and the selector species. Our method may also be applicable for screening intra-species gene-associated polymorphism. Only in that objective the ISSH method may be compared with the In-Gel Competitive Reassociation and EST Array Hybridization method [29], which exploits the property that the vast majority of RFLP fragments between two strains or populations share the same electrophoretic size. Deviation from this property generates false positives and, therefore, the method of Gotoh and Oishi (2003) looses its interest if more distantly related groups are used.

Interestingly, the ISSH method can also be used for isolating the fraction of highly conserved genes between species. This is achieved by rescuing the fraction of ss cDNA that disanneal only at very stringent conditions, which guarantees an almost perfect complementation between the probed and selector pool of transcripts. Moreover, the species from which the selector pool of mRNA is extracted may be selected in order to increase the level of conservation of the enriched cDNA library: the more evolutionary distant the selector species will be, the more conserved the isolated transcripts will be.

The ISSH method is not linked to a specific sequencing technology. In this study we used the long-read 454 FLX technology (Roche) to ensure a minimum sequence length for downstream sequence analyses. However, this argument is currently less valid as the Illumina short-read sequencing technology, which produces many more reads at a lower cost per base, has been recently shown to be useful and accurate in de novo transcriptome assembly of non-model organisms [30]. Traditional Sanger sequencing can also be used providing that the PCR amplified fast evolving transcripts are cloned before sequencing.

\section{Conclusions}

We demonstrated that the ISSH method efficiently enriches a cDNA library in fast evolving genes. As this new method does not rely on the previous knowledge of sequence information, it can be performed on every non-model organism, and is therefore of wide use. Although the improvements and reduced cost of nextgeneration sequencing technologies may lead to ever more complete transcriptomes assemblies, and may have the potential to be used for identifying fast evolving transcripts with bioinformatic tools, the ISSH method will still have and interesting role to play. First, the ISSH method is inexpensive, of little labor, and leads directly to the set of transcripts of interest. Second, as the fast evolving genes are often expressed at low level, they may be hard to retrieve using next-generation sequencing technologies unless very deep sequence coverage is performed, at high cost. Therefore, the ISSH method opens new possibilities in screening transcriptomes in search of genes involved in lineage specific processes and traits, a field of growing interest in evolutionary biology.

\section{Methods}

\section{RNA extraction and preparation of the control library}

Total RNA was extracted from fresh brain tissue of Ancistrus temminckii (probed species) and its close relative Ancistrus dolichopterus (selector species) using TRIzol reagent (Gibco). We also extracted total RNA from our catfish outgroup reference Hypostomus gr. plecostomus. After quantification and quality verification of the total RNA, mRNA was isolated using the mRNA 
Isolation Kit (Roche Diagnostics). The SuperScript double-stranded cDNA synthesis kit (Invitrogen) was used to prepare the brain control library of Ancistrus temminckii and also the outgroup reference Hypostomus gr. plecostomus, starting with $1 \mu \mathrm{g}$ of brain mRNA and the option of oligo(dT) anchor priming for the first strand synthesis step.

\section{The ISSH protocol}

The selector pool of mRNA, extracted in this work from Ancistrus dolichopterus, is biotinylated to allow subsequent separation by magnetic particles coated with streptavidin. Biotinylation of $5 \mu \mathrm{g}$ mRNA was done using the BIO-ULS labeling kit (Kreatech); the final volume was reduce to $7 \mu \mathrm{l}$ using a Speedvac concentrator. The probed pool of mRNAs extracted from the species of interest Ancistrus temminckii is reversetranscribed into ss cDNA using a short-tailed random hexamer primer (5'-AGGA-(N)6-3'). We used $1 \mu \mathrm{g}$ of mRNA (one fifth of the selector's mRNA amount) and $200 \mathrm{ng}$ of the short-tailed random primer in a total volume of $12 \mu \mathrm{l}$. The reverse transcription was performed using the SuperScript II RT (Invitrogen) following the manufacturer's protocol for random priming; the final volume was $20 \mu \mathrm{l}$. The RNA template is destroyed by alkaline hydrolysis $(0.35 \mathrm{~N}$ $\mathrm{NaOH}$; $0.35 \mathrm{M}$ EDTA) at $65^{\circ} \mathrm{C}$ for $15 \mathrm{~min}$. The solution is then neutralized with $0.35 \mathrm{~N} \mathrm{HCl}$ and first strand cDNAs are purified using the Mini Elute PCR Purification Kit (Qiagen) following the manufacturer's protocol but with an additional washing step and two rounds of elution. The final volume was reduced to 7 $\mu \mathrm{l}$ using a Speedvac concentrator.

\section{Inter-Specific Selective Hybridization}

The pool of biotinylated selector mRNA $(7 \mu \mathrm{l})$ and the pool of first strand cDNA of the species of interest $(7 \mu \mathrm{l})$ are mixed and the total volume is adjusted to 15 $\mu \mathrm{l}$. An equal volume $(15 \mu \mathrm{l})$ of $2 \times$ hybridization buffer is added (10 mM EDTA pH8, $1.5 \mathrm{M} \mathrm{NaCl}, 40 \mathrm{mM}$ sodium phosphate buffer pH 7.2, 10× Denhardt's, $0.2 \%$ SDS). The solution is heated at $90^{\circ} \mathrm{C}$ for $2 \mathrm{~min}$ and quickly placed in a rotary shaker located inside a preheated hybridization oven at $55^{\circ} \mathrm{C}$. The hybridization is carried on during 60 hours at $55^{\circ} \mathrm{C}$. At the end of the hybridization step, $75 \mu \mathrm{l}$ of $\mathrm{NaCl} 1 \mathrm{M}$ is added to the hybridization mixture, which is kept at RT.

\section{Separation of the fraction enriched in fast evolving cDNAs}

The selector-probed hybridization mix is sequentially denatured to separate two fractions of cDNAs with increasing denaturation stringencies, the first fraction containing the non-hybridized or non-specifically hybridized probed cDNAs and the second fraction is the one enriched in fast evolving transcripts. First, streptavidin magnetic particles (Roche Diagnostics) are prepared according to the manufacturer's instructions $(1200 \mu \mathrm{g})$ and resuspended in $100 \mu \mathrm{l}$ of TEN 1000 buffer. The hybridization mixture is then transferred in to the tube containing the streptavidin magnetic particles and placed in a rotary shaker for $45 \mathrm{~min}$ at RT. In this step the biotinylated selector mRNAs, which may be hybridized or not with a complementary probed ss cDNA, are linked to the streptavidin magnetic particles. The non-hybridized probed cDNAs are discarded by placing the tube in a magnetic separator (Qiagen) and by removing the supernatant. The magnetic particles with their attached molecules are washed three times at $55^{\circ} \mathrm{C}$ for $15 \mathrm{~min}$, in $600 \mu \mathrm{l}$ of preheated $5 \times \mathrm{SSC}$, then resuspended in $50 \mu \mathrm{l}$ of $0.1 \times \mathrm{SSC}$ and incubated at $65^{\circ} \mathrm{C}$ for $15 \mathrm{~min}$. In this last step the fast evolving probed cDNAs will disanneal from their selector counterpart and this fraction of interest is recovered in the supernatant after a magnetic separation. This step is repeated once. The fraction enriched in fast evolving cDNAs is purified by ethanol precipitation in presence of ammonium acetate and glycogen. The pellet is rinsed once in $70 \%$ ethanol and resuspended in $20 \mu \mathrm{l}$ water.

\section{Second strand synthesis and adapter ligation}

The ss cDNAs are transformed into double stranded (ds) cDNAs using short-tailed random hexamer primers (CCAC-(N)6) and the DNA polymerase I Klenow fragment (Promega), according to the manufacturer's random priming protocol. cDNAs are then blunt ended using T4 DNA Polymerase (Promega), extracted with phenol/chloroform/isoamylalcohol (25:24:1) and recovered by ethanol precipitation with ammonium acetate. Double strand EcoRI adapters (Invitrogen) are ligated to the ds cDNA ends according to the manufacturer's instructions. The final volume is adjusted to $100 \mu \mathrm{l}$ with water and the cDNAs are purified using the High Pure PCR Product Purification Kit (Roche Diagnostics).

\section{PCR amplification of the fraction enriched in fast evolving CDNAs}

A first PCR amplification is performed using a single primer (5'-GTCGACGCGGCCGCGAATT-3') targeted toward the EcoRI adapter ligated at both ends. The PCR reaction is done in $50 \mu$ l final volume with $10 \mu \mathrm{l}$ of cDNA as template and with the following profile: $1 \mathrm{~min}$ initial denaturation at $94^{\circ} \mathrm{C}$ followed by 35 cycles with $30 \mathrm{~s}$ at $94^{\circ} \mathrm{C}, 30 \mathrm{~s}$ at $62^{\circ} \mathrm{C}, 2.5 \mathrm{~min}$ at $72^{\circ} \mathrm{C}$ and a final elongation step of $5 \mathrm{~min}$ at $72^{\circ} \mathrm{C}$. The PCR product is checked on $1,5 \%$ agarose gel. A nested PCR is performed using specific primers overlapping the end of the EcoRI adapters and the tails of the two short-tailed 
random primers used for the synthesis of the first strand and then for the synthesis of the second strand (EcoRIAGGA: 5'-TCGCGGCCGCGTCGACAGGA-3'; EcoRICCAC: 5'-TCGCGGCCGCGTCGACCCAC-3'). The PCR conditions are as described above but the amount of template DNA is adjusted according to the result of the first PCR. The PCR products are checked on 1,5\% agarose gel and then purified using the High Pure PCR Product Purification Kit (Roche Diagnostics).

\section{High-throughput sequencing}

For the Ancistrus control and the outgroup reference Hypostomus gr. plecostomus, shotgun DNA libraries were prepared with a starting amount of $4 \mu \mathrm{g}$ DNA. The mean fragment size was of about $500 \mathrm{bp}$, obtained using nebulizers and chemicals from the GS DNA Library Preparation Kit (Roche Diagnostics) according to the manufacturer's manual. This step was not needed for the Ancistrus library enriched in fast evolving transcripts as the ISSH method results in a PCR product containing fragmented transcripts, generally in the range of 300 to $1000 \mathrm{bp}$. After DNA purification, the DNA end repair step and the ligation of the barcoding adaptors were performed following established protocols [31]. The adapter-ligated DNA from each of the three libraries were pooled and prepared for the 454 sequencing according to standard protocols [32], using the GS DNA Library Preparation Kit with Titanium reagents, and following the instructions of the GS FLX manuals (Roche Diagnostics). The library was sequenced on one 16th region of a full GS FLX sequencing plate with a prior titration run. Upon completion, sequences were screened for primer concatemers, week signal, poly A/T sequences, and barcodes for assigning them to one of the three samples. The average lengths of the reads were $180 \mathrm{bp}$. cDNA assemblies were performed with the SeqMan software from DNAStar. The cDNA library enriched in fast evolving genes and the control library of Ancitrus temminkii were deposited in the Short Read Archive (SRA) of NCBI under the accession number SRA009346.1

\section{Blast search and transcript annotation}

The Blast search against the zebrafish sequences of all the EMBL sub-divisions (Expressed Sequence Tag; High Throughput cDNA sequencing; High Throughput Genome sequencing; mRNA of Standard; Whole Genome Shotgun) were performed on the Vital-IT high-performance computing facility of the Swiss Institute of Bioinformatics http://www.vital-it.ch. We used blast parameter values suitable for comparing divergent sequences (word size $=7$; match score $=+1$; mismatch score $=-1$; initial penalty for opening a gap $=1$; penalty for extending a gap $=2$ ). The local Blast search against our Hypostomus gr. plecostomus brain EST database was performed using the software blast-2.2.19 developed by NCBI. Perl scripts for parsing the blast outputs were built using Eclipse SDK 3.4.1.

The proportion of contigs with low complexity sequences or sequence repeats was assessed using RepeatMasker open-3.2.8 (Smit, AFA, Hubley, R \& Green, P. RepeatMasker Open-3.0. 1996-2004; http:// www.repeatmasker.org). Transcripts were annotated according to their best Blast hit against Swissprot/Uniprot databases, with a minimal E-score of $10 \mathrm{e}-8$. The translations into the six frames were performed using BCM Search Launcher [33] and blasting was done with Blastp at NCBI. Expected frequency of stop codons in non-coding sequences was calculated by multiplying the three single nucleotide frequencies taken from the sequence data of the corresponding library, and summing the frequency of the three possible stop codons. Gene transcription levels in specific tissues were taken from the Unigene database and are expressed in number of ESTs of the gene under consideration divided by the total ESTs of the tissue library, multiplied by 10'000. Gene ontology classification was performed on Panther (Protein ANalysis THrough Evolutionary Relationships; http://www.pantherdb.org), complemented with ontology information given in Uniprot database. We used only the top categories of the classification hierarchy, as given in Panther. Fast evolving transcripts found in Ancistrus temminckii, Hypostomus gr. plecostomus EST, and in the mRNA reference sequences database of NCBI, restricted to the Teleostei (Blastn threshold E-score $<10 \mathrm{e}-8$ ), were used to asses the sequence divergence. A tentative annotated was given according to the best hit against the mRNA refseq database. For direct comparison purposes, sequence divergence was calculated on the sequence region present in all three taxa. Sequences of the reference fast evolving markers were obtained from GeneBank: Ancistrus brevipinnis COI: EU359402; Hypostomus boulengeri COI: EU359422; Danio rerio complete genome: NC_002333; Ancistrus cirrhosus RTN4 introns: EU817562; Hypostomus boulengeri RTN4 introns: EU817560. The RTN4 introns from Danio rerio were retrieved from Ensembl http://www.ensembl.org/, locus: chromosome: Zv8:1:42092991:42094205:1.

\section{Data access}

Raw read data is available at the Short Read Archive (SRA) of NCBI under the accession number SRA009346.1

No ethical approval was required for this study.

\section{Acknowledgements}

We thank Dr. L. Falquet who performed the Blast calculations against the zebrafish transcript databases on the Vital-IT high-performance computing 
facility of the Swiss Institute of Bioinformatics http://www.vital-it.ch. We acknowledge P.-Y. Pettina and A. Coulot for their help in preliminary laboratory tests of feasibility. We thank Dr. Y. Surget-Groba for bioinformatic advises and for revising the manuscript, and two anonymous reviewers for helpful comments and suggestions. This work was supported by funds from the Canton de Genève; the Swiss National Research Fund [grant number 3100A0-122303/1]; and the G \& A Claraz Foundation.

\section{Authors' contributions}

$J I M B$ conceived the project and designed the analyses. JIMB and AF performed the bioinformatic and sequence analyses. JIMB and IB performed the experimental work. The manuscript was prepared by JIMB with assistance from AF and IB. All authors have read and approved the final version of the manuscript.

Received: 24 September 2009

Accepted: 22 February 2010 Published: 22 February 2010

\section{References}

1. Cardoso YP, Montoya-Burgos Jl: Unexpected diversity in the catfish Pseudancistrus brevispinis reveals dispersal routes in a Neotropical center of endemism: the Guyanas Region. Mol Ecol 2009, 18(5):947-964.

2. Chiachio MC, Oliveira C, Montoya-Burgos Jl: Molecular systematic and historical biogeography of the armored Neotropical catfishes Hypoptopomatinae and Neoplecostominae (Siluriformes: Loricariidae). Mol Phylogen Evol 2008, 49(2):606-617.

3. Montoya-Burgos Jl: Historical biogeography of the catfish genus Hypostomus (Siluriformes: Loricariidae), with implications on the diversification of Neotropical ichthyofauna. Mol Ecol 2003, 12(7):1855-1867.

4. Sunnucks P: Efficient genetic markers for population biology. Trends Ecol Evol 2000, 15(5):199-203.

5. Tripathi N, Hoffmann M, Willing EM, Lanz C, Weigel D, Dreyer C: Genetic linkage map of the guppy, Poecilia reticulata, and quantitative trait loci analysis of male size and colour variation. Proc R SOC B 2009, 276(1665):2195-2208.

6. Inoue $\mathrm{S}$, Kiyama R, Oishi M: Construction of highly extensive polymorphic DNA libraries by in-gel competitive reassociation procedure. Genomics 1996, 31(3):271-276.

7. Nicod JC, Largiader CR: SNPs by AFLP (SBA): a rapid SNP isolation strategy for non-model organisms. Nucleic Acids Res 2003, 31(5):e19.

8. Garrick R, Sunnucks P: Development and application of three-tiered nuclear genetic markers for basal Hexapods using single-stranded conformation polymorphism coupled with targeted DNA sequencing. BMC Genet 2006, 7(1):11.

9. Pollard KS, Salama SR, Lambert N, Lambot MA, Coppens S, Pedersen JS, Katzman S, King B, Onodera C, Siepel A, et al: An RNA gene expressed during cortical development evolved rapidly in humans. Nature 2006, 443(7108):167-172.

10. Ali F, Meier R: Positive Selection in ASPM Is Correlated with Cerebral Cortex Evolution across Primates but Not with Whole-Brain Size. Mol Biol Evol 2008, 25(11):2247-2250.

11. Orr HA, Masly JP, Presgraves DC: Speciation genes. Curr Opin Genet Dev 2004, 14(6):675-679.

12. Schluter D, Conte GL: Genetics and ecological speciation. Proc Natl Acad Sci USA 2009, 106:9955-9962.

13. Haerty W, Jagadeeshan S, Kulathinal RJ, Wong A, Ravi Ram K, Sirot LK, Levesque L, Artieri CG, Wolfner MF, Civetta A, et al: Evolution in the Fast Lane: Rapidly Evolving Sex-Related Genes in Drosophila. Genetics 2007, 177(3):1321-1335.

14. Karn RC, Clark NL, Nguyen ED, Swanson WJ: Adaptive Evolution in Rodent Seminal Vesicle Secretion Proteins. Mol Biol Evol 2008, 25(11):2301-2310.

15. Moreno-Estrada A, Tang K, Sikora M, Marques-Bonet T, Casals F, Navarro A, Calafell F, Bertranpetit J, Stoneking M, Bosch E: Interrogating eleven fastevolving genes for signatures of recent positive selection in worldwide human populations. Mol Biol Evol 2009, msp134.

16. Brcic-Kostic K: Neutral mutation as the source of genetic variation in life history traits. Genet Res 2005, 86(1):53-63.

17. Schmid KJ, Tautz D: A screen for fast evolving genes from Drosophila. Proc Natl Acad Sci USA 1997, 94(18):9746-9750.
18. Clark AG, Eisen MB, Smith DR, Bergman CM, Oliver B, Markow TA, Kaufman TC, Kellis M, Gelbart W, lyer VN, et al: Evolution of genes and genomes on the Drosophila phylogeny. Nature 2007, 450(7167):203-218.

19. Nielsen R, Bustamante C, Clark AG, Glanowski S, Sackton TB, Hubisz MJ, Fledel-Alon A, Tanenbaum DM, Civello D, White TJ, et al: A scan for positively selected genes in the genomes of humans and chimpanzees. PLOS Biol 2005, 3(6):976-985.

20. Barbash DA, Siino DF, Tarone AM, Roote J: A rapidly evolving MYB-related protein causes species isolation in Drosophila. Proc Natl Acad Sci USA 2003, 100(9):5302-5307.

21. Rowekamp W, Firtel RA: Isolation of developmentally regulated genes from Dictyostelium. Dev Biol 1980, 79(2):409-418.

22. Schutzbank T, Robinson R, Oren M, Levine AJ: SV40 large tumor antigen can regulate some cellular transcripts in a positive fashion. Cell 1982, 30(2):481-490.

23. Scott MR, Westphal KH, Rigby PW: Activation of mouse genes in transfromed cells. Cell 1983, 34(2):557-567.

24. Subramanian S, Kumar S: Gene expression intensity shapes evolutionary rates of the proteins encoded by the vertebrate genome. Genetics 2004 168(1):373-381.

25. Zhang LQ, Li WH: Mammalian housekeeping genes evolve more slowly than tissue-specific genes. Mol Biol Evol 2004, 21(2):236-239.

26. Tuller $T$, Kupiec $M$, Ruppin E: Evolutionary rate and gene expression across different brain regions. Genome Biology 2008, 9(9):R142.

27. Eisenberg E, Levanon EY: Human housekeeping genes are compact. Trends Genet 2003, 19(7):362-365.

28. Carninci P, Kasukawa T, Katayama S, Gough J, Frith MC, Maeda N, Oyama R, Ravasi $T$, Lenhard B, Wells $C$, et al: The transcriptional landscape of the mammalian genome. Science 2005, 309(5740):1559-1563.

29. Gotoh K, Oishi M: Screening of gene-associated polymorphisms by use of in-gel competitive reassociation and EST (CDNA) array hybridization. Genome Res 2003, 13(3):492-495.

30. Gibbons JG, Janson EM, Hittinger $C T$, Johnston $M$, Abbot $P$, Rokas $A$ : Benchmarking Next-Generation Transcriptome Sequencing for Functional and Evolutionary Genomics. Mol Biol Evol 2009, 26(12):2731-2744.

31. Meyer M, Stenzel U, Hofreiter M: Parallel tagged sequencing on the 454 platform. Nature Protocols 2008, 3(2):267-278.

32. Margulies M, Egholm M, Altman WE, Attiya S, Bader JS, Bemben LA, Berka J, Braverman MS, Chen YJ, Chen ZT, et al: Genome sequencing in microfabricated high-density picolitre reactors. Nature 2005, 437(7057):376-380.

33. Smith RF, Wiese BA, Wojzynski MK, Davidson DB, Worley KC: BCM Search Launcher - An integrated interface to molecular biology data base search and analysis services available on the World Wide Web. Genome Res 1996, 6(5):454-462.

doi:10.1186/1471-2164-11-126

Cite this article as: Montoya-Burgos et al:: Transcriptome screen for fast evolving genes by Inter-Specific Selective Hybridization (ISSH). BMC Genomics 2010 11:126.

\section{Submit your next manuscript to BioMed Central and take full advantage of:}

- Convenient online submission

- Thorough peer review

- No space constraints or color figure charges

- Immediate publication on acceptance

- Inclusion in PubMed, CAS, Scopus and Google Scholar

- Research which is freely available for redistribution

Submit your manuscript at www.biomedcentral.com/submit
C Biomed Central 